Departamento de Ciência e Tecnologia, Secretaria de Ciência, Tecnologia e Insumos Estratégicos, Ministério da Saúde

Correspondência | Correspondence: Decit - Departamento de Ciência e Tecnologia do Ministério da Saúde Esplanada dos Ministérios Bloco G sala 845 70058-900 Brasília, DF, Brasil

Texto de difusão técnico-científica do Ministério de Saúde.

\section{Fortalecendo a pesquisa clínica no Brasil: a importância de registrar os ensaios clínicos}

\section{Strengthening clinical research in Brazil: the importance of registering clinical trials}

Dentre as diversas definições disponíveis na literatura para o termo "pesquisa clínica", o manual publicado pelo Departamento de Ciência e Tecnologia (Decit/ SCTIE/MS), resultante da II Conferência Nacional de Ciência e Tecnologia e Inovação em Saúde, a define como: tipo de pesquisa que segue métodos científicos aplicáveis aos seres humanos - denominados voluntários ou "sujeitos da pesquisa" - sadios ou enfermos, de acordo com o objetivo da pesquisa.

Segundo a Association of American Medical Colleges Task Force on Clinical Research, a pesquisa clínica é definida como investigação na área da saúde destinada à produção de conhecimentos essenciais para a compreensão dos mecanismos, prevenção e tratamento de doenças, assim como à promoção da saúde.

AAgência Européia para Avaliação de Produtos Medicinais define pesquisa clínica como "investigação em seres humanos, com o objetivo de descobrir ou verificar os efeitos farmacodinâmicos, farmacológicos, clínicos e/ou outros efeitos de produto(s) e/ou identificar reações adversas ao(s) produto(s) em investigação, com o objetivo de averiguar sua segurança e/ou eficácia”.

Uma proposta também discutida é o uso do termo "Pesquisa Clínica Ampliada," cujas atividades teriam como objetivo o desenvolvimento de novos conhecimentos e tecnologias de prevenção e diagnóstico de doenças, de tratamento e reabilitação de doentes. Entre essas atividades, estão inovações na prestação de serviços de atenção e vigilância em saúde; análises antropológicas; novas abordagens das clínicas complementares no atendimento, na vigilância, na reabilitação; avaliações interdisciplinares de doenças emergentes, entre outras. ${ }^{\mathrm{a}}$

As várias definições existentes para o termo "pesquisa clínica" corroboram para classificações diversas nos bancos de dados de pesquisa no Brasil. Por meio de busca realizada no sítio Pesquisa Saúde, Base de Dados Gerencial do Decit ${ }^{b}$, empregando-se o filtro Subagenda - Pesquisa Clínica, encontraram-se 240 projetos de pesquisa e um total de R $\$ 126$ milhões investidos nesses projetos no período de 2002 a 2009 (Tabela). Esses projetos foram classificados como pesquisa clínica pelo próprio coordenador do estudo.

Dos 240 projetos registrados na base de dados Pesquisa Saúde, 226 foram reclassificados pela equipe da área de pesquisa clínica do Decit em seis categorias: 80 projetos de pesquisa básica, 46 estudos pré-clínicos, 37 pesquisas clínicas excluindo ensaios clínicos, 38 ensaios clínicos, 24 projetos de infra-estrutura em pesquisa clínica e um projeto de avaliação tecnológica em saúde. Quatorze projetos não tinham informação suficiente e não puderam ser classificados. Todos os projetos são apoiados pelo Decit/SCTIE/MS com a parceria financeira do Conselho Nacional de Desenvolvimento Científico e Tecnológico (CNPq), Financiadora de Projetos e Pesquisas (Finep), Fundações de Amparo à Pesquisa, Secretarias Estaduais de Saúde e de Ciência e Tecnologia.

Para essa classificação dos projetos, utilizaram-se os conceitos de pesquisa clínica mencionados; para as outras classificações, utilizaram-se as definições a seguir.

De acordo com o manual Frascati, elaborado pela Organização para a Cooperação e o Desenvolvimento Econômico, a pesquisa básica é o estudo teórico ou experimental que contribui para a compreensão sobre os fatos e fenômenos observáveis, teorias, sem ter em vista uso ou aplicação específica imediata. É comumente executada por cientistas que estabelecem suas próprias metas e organizam o seu próprio trabalho. Contudo, em alguns casos, a pesquisa básica pode ser fundamentalmente orientada ou dirigida em função de áreas mais amplas de interesse geral. 
Tabela. Fomento do Ministério da Saúde e parceiros a projetos da subagenda de pesquisa clínica. Brasil, 2002-2009

\begin{tabular}{lcccc}
\hline Modalidade de fomento & Fomento descentralizado & Fomento nacional & Contratação direta & Total \\
\hline Projetos & 68 & 162 & 10 & 240 \\
Total de recursos & $\mathrm{R} \$ 7.386 .959,50$ & $\mathrm{R} \$ 115.193 .138,54$ & $\mathrm{R} \$ 4.049 .590,47$ & $\mathrm{R} \$ 126.629 .688,51$ \\
\hline
\end{tabular}

Fonte: Dados do Departamento de Ciência e Tecnologia (Decit/SCTIE/MS)

Os estudos pré-clínicos, de acordo com a Agência Nacional de Vigilância Sanitária, correspondem à aplicação de uma nova molécula em animais, após ser identificada em experimentações in vitro como tendo potencial terapêutico. São obtidas informações preliminares sobre a atividade farmacológica e segurança da nova molécula estudada. Nessa fase, mais de $90 \%$ das substâncias estudadas são eliminadas por não demonstrarem suficiente atividade farmacológica/terapêutica ou serem consideradas demasiadamente tóxicas.

Os ensaios clínicos são realizados majoritariamente no processo de desenvolvimento de novos medicamentos e são classificados em quatro fases (I a IV), conforme definido pela Resolução n ${ }^{\circ}$ 251/97 do Conselho Nacional de Saúde. A fase I é o primeiro estudo, em seres humanos, em pequenos grupos de pessoas voluntárias, em geral sadias, de um novo princípio ativo ou nova formulação para estabelecer uma evolução preliminar da segurança e do perfil farmacocinético. A fase II visa demonstrar a atividade e estabelecer a segurança em curto prazo do princípio ativo, avaliando a relação dose-resposta em um número limitado de pacientes enfermos. A fase III é realizada em grandes e variados grupos de pacientes para determinar o resultado do risco/benefício a curto e longo prazos das formulações do princípio ativo, explorando-se o tipo e perfil das reações adversas mais freqüentes. A fase IV são pesquisas realizadas depois de comercializado o produto e/ou especialidade medicinal.

A pesquisa clínica brasileira teve um crescimento significativo, constatada por meio do número de registros de ensaios clínicos brasileiros em base de dados estrangeira, como o Clinical Trials, sítio americano de registro de pesquisa clínica. De 187 registros de estudos brasileiros em 2005 (184 de intervenção e três observacionais), em 2010 passou a 414 registros (351 de intervenção e 63 observacionais).

A Figura 1 mostra a proporção de projetos e de recursos investidos por região geográfica do Brasil e a Figura 2, a proporção de projetos e recursos distribuídos por ano. Os indicadores por região mostram maior proporção de projetos na região sudeste, o que se justifica pela tradição de pesquisa e grande número de investigadores nessa região. A maior proporção de projetos em 2005 coincide com a criação da Rede Nacional de Pesquisa Clínica (RNPC), cujo objetivo é a consolidação da pesquisa clínica no Brasil.

A franca expansão da pesquisa clínica no Brasil coincide, não por acaso, com as ações tomadas pelo Ministério da Saúde, como o financiamento de infra-estrutura e criação da RNPC. A rede foi criada em 2005 por meio de uma iniciativa do Ministério da Saúde, co-financiada pelo Ministério da Ciência e Tecnologia (MCT) e pela Financiadora de Estudos e Projetos (Finep). A criação da RNPC mostra o empenho governamental conferido às atividades científicas e tecnológicas em saúde no País. $\mathrm{O}$ desenvolvimento de trabalho em rede segue a orientação constitucional apoiada no tripé da universalidade, integralidade e eqüidade. Além disso, exige o efetivo comprometimento das equipes de pesquisa clínica com as prioridades fixadas na Política Nacional de Saúde.

Atualmente, a RNPC é formada por 32 centros de pesquisa clínica ligados às instituições de ensino superior distribuídos pelas cinco regiões geográficas do País (Figura 3). Nesses cinco anos, desde a sua criação, a rede tem favorecido a cooperação e o intercâmbio entre os pesquisadores, ações que potencializam a obtenção de resultados significativos na produção de conhecimento.

A estratégia governamental ao criar a rede visou, prioritariamente, fomentar a execução de todas as fases de ensaios clínicos de fármacos, procedimentos, equipamentos, dispositivos médicos e novos métodos de diagnósticos no Brasil. A RNPC desenvolve várias ações de fomento à pesquisa e contribui para a formação de recursos humanos, por meio da realização de cursos de capacitação em pesquisa clínica. A aproximação

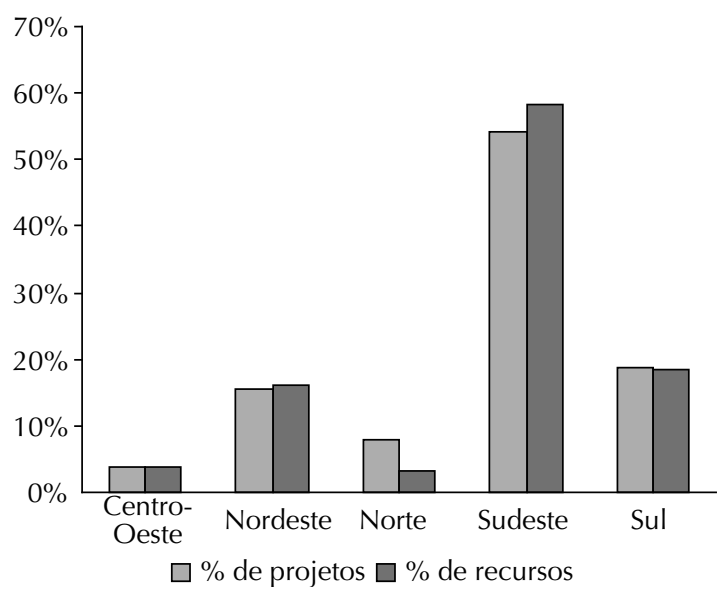

Fonte: Dados do Departamento de Ciência e Tecnologia (Decit/SCTIE/MS)

Figura 1. Fomento do Ministério da Saúde e parceiros a projetos da subagenda de pesquisa clínica segundo a região. Brasil, 2002-2009. 


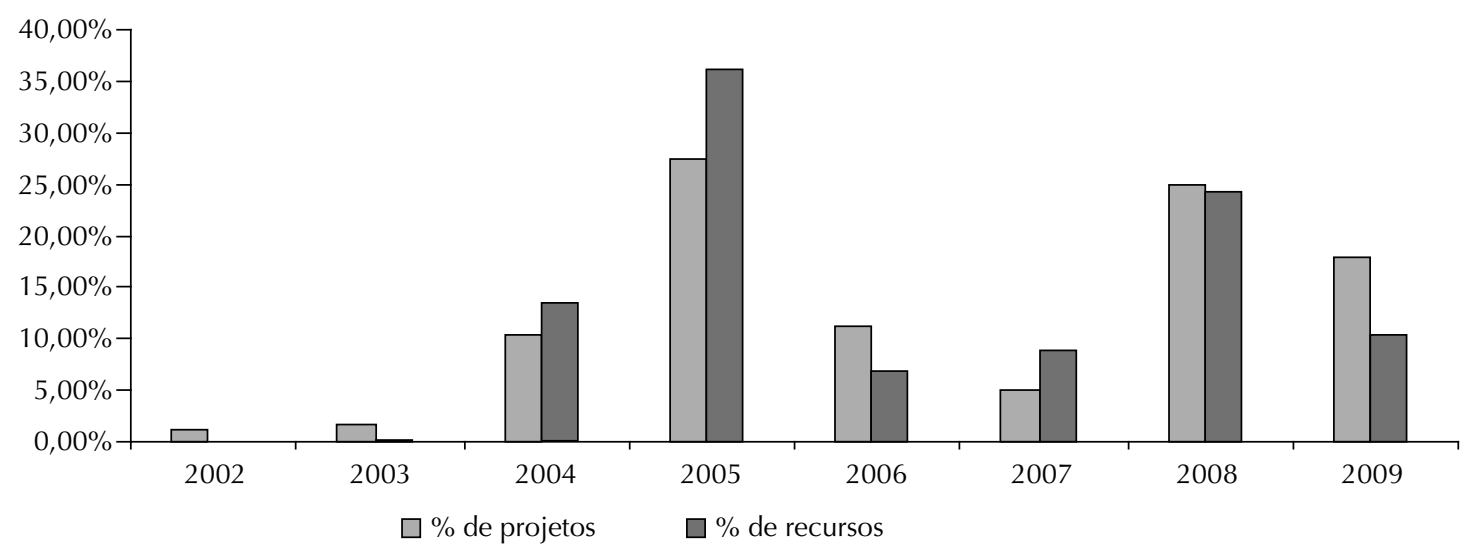

Fonte: Dados do Departamento de Ciência e Tecnologia (Decit/SCTIE/MS)

Figura 2. Fomento do Ministério da Saúde e parceiros a projetos da subagenda de pesquisa clínica segundo o ano. Brasil 2002-2009.

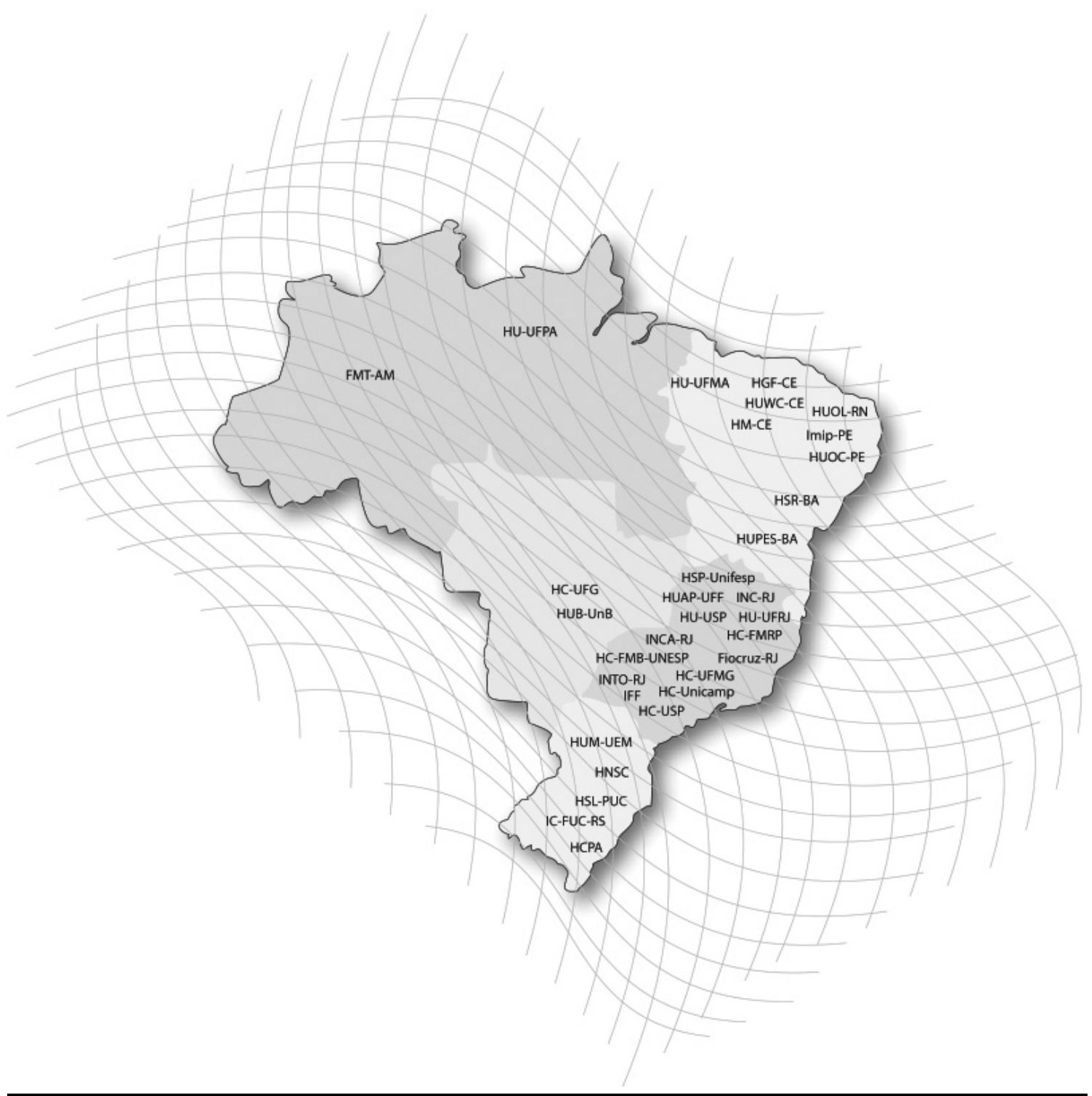

Figura 3. Distribuição geográfica dos centros da RNPC no Brasil. 
dos centros de pesquisa da RNPC permite propiciar a transferência de tecnologia e de conhecimentos entre os centros de pesquisa da rede e desenvolver estudos multicêntricos de relevância para o desenvolvimento técnico-científico do País. Os estudos em desenvolvimento na RNPC incluem temas como: apnéia obstrutiva do sono, osteoporose grave, hipertensão arterial, hipertensão resistente, leishmanioses, hanseníase, cirurgia bariátrica, entre outros.

\section{REGISTRO BRASILEIRO DE ENSAIOS CLÍNICOS - REBEC}

Simultaneamente à evolução da pesquisa clínica no Brasil foi criado o Rebec, ${ }^{\text {c }}$ cujo objetivo é proporcionar aos pacientes, médicos, investigadores, comitês de ética e patrocinadores acesso a informações sobre os ensaios clínicos em andamento e concluídos, evitando a duplicação de esforços e impulsionando o avanço do conhecimento e a cooperação entre grupos de pesquisa. A criação da referida plataforma, lançada em dezembro de 2010, é resultado do esforço conjunto do Ministério da Saúde, do Instituto de Comunicação e Informação Científica e Tecnológica em Saúde (Icict/Fiocruz), da Organização Pan-americana de Saúde (Opas) e do Centro Latino-americano e do Caribe de Informação em Ciências da Saúde (Bireme).

O registro do ensaio clínico em bases de dados públicas permite o acesso à informação sobre os efeitos de um produto ou procedimento em investigação. Por conseguinte, contribui para a transparência na pesquisa, pelo fortalecimento dos valores éticos que envolvem os estudos científicos em seres humanos.

Tendo em vista a relevância da pesquisa clínica, o Rebec inclui ensaios clínicos que avaliam intervenções em saúde resultantes do uso de medicamentos, células e outros produtos biológicos, procedimentos cirúrgicos e radiológicos, dispositivos médicos, terapias comportamentais e complementares, mudanças no processo de atenção preventiva, entre outros.

O Rebec propicia à sociedade uma plataforma livre e gratuita para o registro de ensaios clínicos envolvendo seres humanos que permite o acesso via Internet aos dados de todas as pesquisas em andamento, independentemente da publicação dos resultados. A existência de um registro brasileiro garante acesso da sociedade às informações em língua portuguesa sobre o estágio de desenvolvimento dos estudos no Brasil. Da mesma forma, o Rebec favorece o acesso de voluntários às pesquisas em andamento.

O Rebec está preparado para se integrar à International Clinical Trials Registry Platform da Organização Mundial da Saúde (ICTRP/OMS). A ICTRP reúne dados de uma rede de 11 registros primários internacionais, permitindo consultas a uma base de dados unificada na Internet. ${ }^{\mathrm{d}} \mathrm{O}$ processo de certificação será concluído no primeiro semestre de 2011, quando também os dados brasileiros estarão disponíveis para consulta no sítio da ICTRP.

O sistema foi desenhado para comportar registros em línguas portuguesa, inglesa e espanhola. Podem ser registrados estudos nacionais, estudos multicêntricos internacionais com sítios de pesquisa no Brasil e estudos internacionais, embora neste caso seja desejável o registro em plataforma cujo idioma principal seja o mesmo do país em que o estudo se desenvolve.

O Rebec propicia uma importante fonte de informação para os pacientes, facilitando seu recrutamento, como também para os profissionais de saúde, pesquisadores, empresas e centros de pesquisa, propiciando maior interação e inserção internacional.

\section{COMENTÁRIOS FINAIS}

A criação da RNPC em hospitais de ensino juntamente com o Rebec representam ações do Ministério da Saúde que contribuem concretamente para o desenvolvimento da pesquisa clínica no Brasil. A estratégia governamental reuniu os parceiros necessários para a construção de um novo modelo de pesquisa clínica, voltado às reais necessidades do Sistema Único de Saúde, visando à consolidação da rede a fim de executar todas as fases de ensaios clínicos no Brasil.

O Rebec possui como vantagens: redução da duplicação desnecessária de esforços de pesquisa ao informar os profissionais que planejam novos estudos acerca de todos os ensaios existentes; possibilidade de aumentar as taxas de recrutamento de participantes para ensaios clínicos (especialmente para doenças raras ou condições de alto risco); agregar valor aos resultados da pesquisa ao prover uma fonte de informação confiável sobre ensaio clínico para estudos (revisões sistemáticas, metanálises e diretrizes) baseados em evidências e aprimorar as evidências disponíveis que orientem as práticas de saúde.

\footnotetext{
c Ministério da Saúde. Registro Brasileiro de Ensaios Clínicos [citado 16 fev 2011]. Disponível em: http://www.ensaiosclinicos.gov.br ${ }^{d}$ World Health Organization. International Clinical Trials Registy Plataform. Geneva; [s.d.] [citado 16 fev 2011]. Disponível em: http:// www.who.int/ictrp
} 